\title{
Vertical Micromirrors Integrated With Electromagnetic Microactuators for Two-Dimensional Optical Matrix Switches
}

\author{
Guo-Dung John Su, Member, IEEE, Chen-Wei Chiu, and Fukang Jiang
}

\begin{abstract}
We report novel single crystalline silicon (SCS) micromirrors vertically attached to electromagnetic microactuators for the two-dimensional (2-D) free-space optical cross-connect switching application. The operation voltage is typically $0.7 \mathrm{~V}$ due to the large electromagnetic force generated by the coils on the microactuators. Vertical micromirrors with the surface area of $500 \times 1200 \mu \mathrm{m}$ are monolithically integrated with microactuators. The micromirrors are fabricated by low-cost tetra-methyl ammonium hydroxide anisotropic wet etching on the (110) SCS wafers. The surface roughness of the micromirror is less than $20 \mathrm{~nm}$ and the measured insertion loss caused by the micromirror is approximately $0.2 \mathrm{~dB}$. The large surface area of the micromirror can accommodate a big optical beam for 2-D matrix configuration to minimize insertion loss. Chips consisting of $10 \times 10$ arrays are successfully fabricated and tested.
\end{abstract}

Index Terms-Electromagnetic force, free space, low voltage, optical cross-connect switches, optical microelectromechanical systems (MEMS), vertical micromirrors.

\section{INTRODUCTION}

$\mathbf{T}$ HE HIGH bandwidth demand in communication has stimulated extensive research activity in expanding the capacity of optical telecommunication networks. Over the past few years, microelectromechanical systems (MEMS) technology has emerged as a promising candidate to achieve true all-optical wavelength routing [1]-[4] for the high bandwidth requirement. Leveraging inherent advantages of MEMS devices such as batch fabrication process, small size, and scalability, MEMS optical switches could provide reliable, repeatable, and fast switching functions, compared with traditional optomechanical switches. More importantly, MEMS technology enables large matrix switches to be monolithically fabricated on a single chip, which makes it is possible to build large-scale optical cross-connect switches for future optical communication networks.

Two-dimensional (2-D) configuration of MEMS optical switches [5]-[8] is known for the merits of easy fabrication, simple control scheme, and high reliability. Collimated optical beams are usually employed in free-space MEMS optical

\footnotetext{
Manuscript received March 30, 2005; revised May 8, 2005. This work was supported in part by National Science Council of Taiwan under NSC 93-2218-E$002-135$.

G.-D. J. Su and C.-W. Chiu are with the Graduate Institute of ElectroOptical Engineering, National Taiwan University, Taipei 106, Taiwan (e-mail: gdjsu@cc.ee.ntu.edu.tw; chenweic@yahoo.com).

F. Jiang is with Umachines, Inc., Altadena, CA 91001 USA (e-mail: fjiang@umachines.com).

Digital Object Identifier 10.1109/LPT.2005.852642
}

switches. A general matrix-type $N \times N$ MEMS optical switch will suffer the noticeable insertion loss due to the optical beam divergence from the finite emitting aperture of fiber collimators. To overcome this difficulty, large micromirrors are desired to accommodate large beam diameters in the order of few hundreds of micrometers [9]. This requires long traveling distance of microactuators and is in favor of electromagnetic actuation force from the point of view of energy density [10]. In other words, the electromagnetic actuation needs much less actuation voltage to move micromirrors than the popular electrostatic method [11]. In addition to longitudinal misalignment above, the angular misalignment and the flatness of micromirrors in MEMS optical switches will add additional optical insertion loss. The angular misalignment can be solved by vertical micromirrors so that the surface normal of micromirrors remains constant as moving the micromirrors in/out the optical path. Regarding the flatness, micromirrors made of single crystalline silicon (SCS) are preferred because SCS was proved stress free and optically flat [12]. Previously, vertical micromirrors are shaped by deep reactive ion etching (DRIE) and smoothed by wet etching [13]. DRIE, however, suffers nonuniform etching rate cross the wafer [14] and the etching depth is limited. On the other hand, chemical wet etching could make uniform and large micromirrors, but it is a nontrivial process and needs careful etching mask designs to avoid lateral etching.

In this letter, we report a novel MEMS optical switch monolithically integrated with vertical SCS micromirrors. The switch is actuated by electromagnetic force and the operating voltage is less than $1 \mathrm{~V}$. The vertical micromirror is shaped and smoothed by tetra-methyl ammonium hydroxide (TMAH) anisotropic wet etching in one step to reduce the fabrication cost and to make high-quality micromirrors. In addition, TMAH is more IC compatible, less toxic, and more stable [15], [16] than other alkaline-based wet etchant, such as potassium hydroxide $(\mathrm{KOH})$. The surface area of micromirrors made by TMAH is $500 \times$ $1200 \mu \mathrm{m}$ and the surface roughness is less than $20 \mathrm{~nm}$ typically. The optical insertion loss caused by the micromirrors is about $0.2 \mathrm{~dB}$. Chips consisting of $10 \times 10$ arrays are successfully fabricated and tested.

\section{DESIGN AND FABRICATION}

A schematic drawing and the operation principle of a switching element is shown in Fig. 1. A vertical SCS mirror made by anisotropic wet etching is attached to the microactuation flap to switch the light into the selected channel. The switch is actuated by the electromagnetic force that is generated 


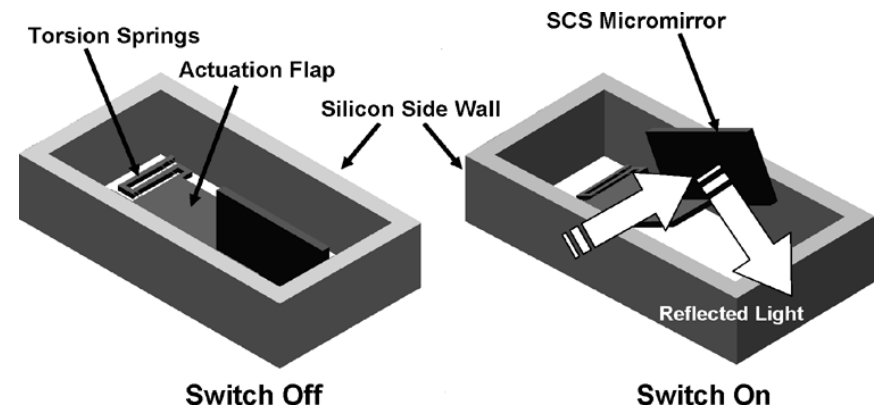

Fig. 1. Schematic drawing of a vertical micromirror for 2-D optical switches.

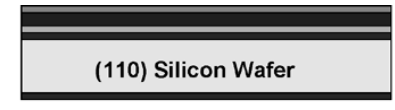

(1). Start with (110) wafer with protection layers

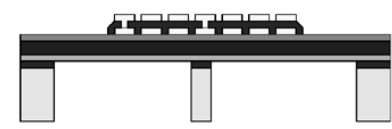

(3). Pattern the metal coils on the top side of the wafer

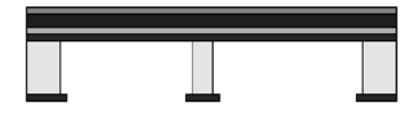

(2). Wet etching on the bottom side of the wafer to form vertical micromirror

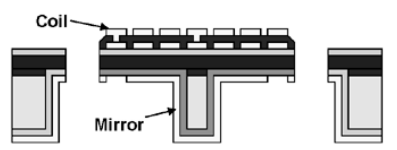

(4). Pattern the actuation flap and torsion springs to release the switching unit
Fig. 2. Fabrication processes of optical MEMS switches monolithically integrated with vertical micromirrors.

by metal coils on the actuation flap and external permanent magnets. The metal coils are patterned on the other side of micromirrors and permanent magnets are attached underneath the actuation flap. When the current is applied to the metal coils on the actuation flap, the electromagnetic force moves the single-crystalline silicon mirror out of the etched silicon cavity to reflect the light. The moving direction of the silicon micromirror is perpendicular to the collimated lights to minimize angular misalignment. The design of the micromirror is $20 \mu \mathrm{m}$ thick, $500 \mu \mathrm{m}$ high, and $1200 \mu \mathrm{m}$ long to ensure low optical insertion loss and crosstalks. Besides, vertical micromirrors could save the precious wafer surface area and the mirror size can be increased without adding light traveling distance, such as surface micromachining switches. In order to eliminate any possible stiction, the design does not use any mechanical stopper in the moving path of the micromirror. The microactuation flap is attached to the silicon sidewall with serpentine springs.

To fabricate MEMS optical switches with smooth and vertical reflecting surfaces, we started with double-sided polished silicon-on-insulator wafers with the (110) substrate and the (100) device layer, which were fusion bonded together. Low temperature oxide was first deposited and patterned as the wet etching masks. Using anisotropic TMAH wet etching, vertical walls were created on the (111) planes in the (110) substrate wafer and were used as the reflecting mirror, as shown in Step 2 of Fig. 2. SCS micromirrors made by wet etching are stress-free and flat due to the crystal orientation. The cost of wet etching is relatively low compared with other MEMS etching processes of making high aspect ration structures, such as DRIE, and the etching results are predictable and repeatable [17], [18].

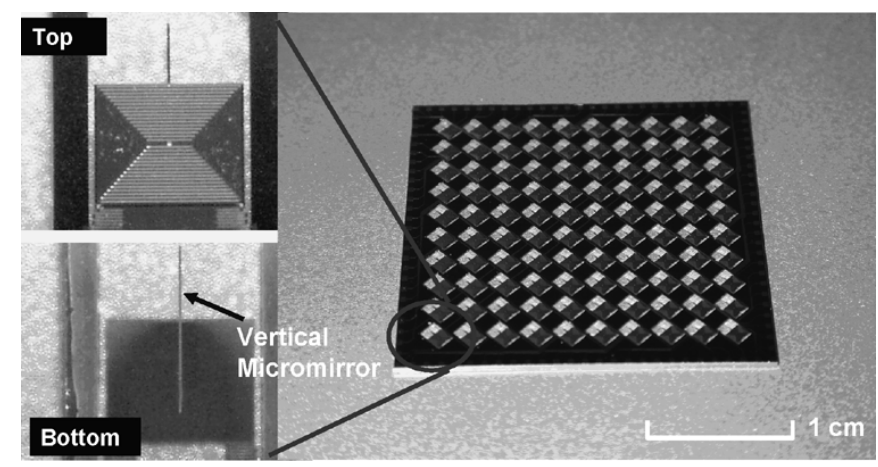

Fig. 3. Pictures of a $10 \times 10$ matrix and a detailed switching unit.

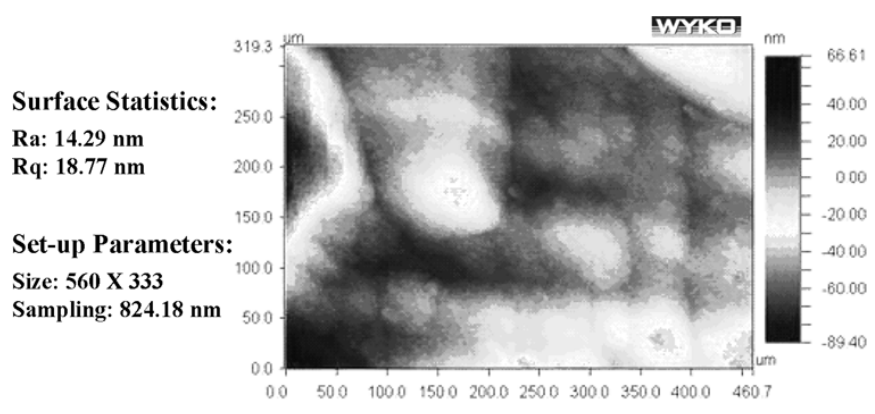

(a)

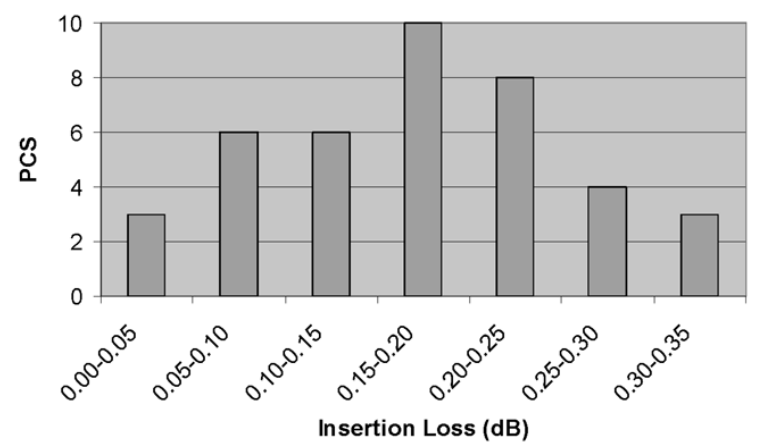

(b)

Fig. 4. (a) Typical surface roughness profiles, and (b) insertion loss measurement of 40 pieces of micromirrors.

After the vertical mirrors were made, the gold was deposited by E-beam evaporator on the device layers and patterned to make metal coils for generating electromagnetic force, as shown in Step 3 of Fig. 2. Then flaps and serpentine springs were patterned by DRIE on the device layer to release microactors. The picture of a $10 \times 10$ switch arrays is shown in Fig. 3 with zoomed pictures of a single switching element.

\section{EXPERIMENTAL TEST RESUlTS AND DISCUSSION}

The required voltage to move the entire micromirror out of the silicon cavity is $0.7 \mathrm{~V}$ with the current of $10 \mathrm{~mA}$ typically. The switching is achieved by passing the current through the metal coils on the actuation flap to move the micromirror up and down. The surface roughness of the SCS micromirror is measured by an optical interferometer made by WYKO. The typical surface profile of wet-etched micromirror is shown in Fig. 4(a). Both the average roughness and root-mean-square roughness are usually 
less than $20 \mathrm{~nm}$. The scattering light caused by the micromirror due to the surface roughness is estimated [6] as

$$
P_{\text {scat }}=\left(1-e^{-\left(\frac{4 \pi \sigma \cos \theta_{i}}{\lambda}\right)^{2}}\right) P_{\text {tot }}
$$

where $P_{\text {tot }}$ is the total flux of incident light, $P_{\text {scat }}$ is the flux of light scattered, $\lambda$ is the wavelength, $\theta_{i}$ is the incident angle, and $\sigma$ is the surface roughness. Assuming that the scattering light accounts for the major optical power loss, the insertion loss of the SCS vertical micromirror etched by TMAT is calculated less than $0.2 \mathrm{~dB}$ at the wavelength of $1.55 \mu \mathrm{m}$.

The optical insertion loss was measured using a pair of commercial single-mode fiber collimators (from NSG) with a GRIN lens attached to a fiber. The GRIN lens is antireflection-coated at $1.55 \mu \mathrm{m}$, and fiber collimators are mounted on the five axis precision alignment stage. The beam diameter $\left(1 / e^{2}\right)$ from the fiber is $200 \mu \mathrm{m}$, which can be almost covered by the vertical micromirror $(500 \times 1200 \mu \mathrm{m})$. The insertion losses of the micromirrors were measured with 40 pieces to study the statistic distribution of the surface quality. It was found that the insertion loss fell into the range of $0.2 \pm 0.15 \mathrm{~dB}$, which is close to the theoretical calculation above. We suspect that the high insertion loss from some micromirrors may be due to impurities in the (110) silicon wafers during the wafer growth.

To achieve high optical coupling efficiencies, the alignment of external optical components to the chip has to be as precise as possible. The surface normal of vertical micromirrors is uniform because it is aligned to the silicon crystal orientation. This reduces the effects of angular misalignment. In addition, insertion loss caused by the surface flatness has been experimentally proved low. We assume that longitudinal misalignment will be a determining factor to optical insertion loss and the coupling efficiency is a function of coupling length. The collimated optical beams diverge as they propagate through free space due the finite emitting beam aperture. This imposes fundamental limits on optical coupling efficiency. By applying Gaussian shaped mode, we noticed that the coupling loss drops rapidly as the mirror and beam size increase, suggesting that the beam divergence is the dominant factor in this region. The effect saturates for the mirror radii larger than $500 \mu \mathrm{m}$. This is based on truncation ration of 0.5 , which means that the waist diameters of Gaussian beam from a fiber collimator is half the size of the micromirror. Fiber-micromirror-fiber losses of $2.5 \mathrm{~dB}$ were demonstrated through the longest path of the $10 \times 10$ switch arrays. The experimental results are seen to agree well with the theoretical analysis.

\section{CONCLUSION}

A novel MEMS optical switch monolithically integrated with a vertical mirror has been demonstrated. The switching voltage is less than $1 \mathrm{~V}$ and the switching time is approximately $6 \mathrm{~ms}$. The switch is actuated by electromagnetic force generated by the coils on the actuators and external permanent magnets. The power consumption is less than $10 \mathrm{~mW}$. A vertical mirror with the surface area of $500 \times 1200 \mu \mathrm{m}$ is etched on the (111) surface of the (110) SCS. The surface roughness of the micromirror is less than $20 \mathrm{~nm}$ and the insertion loss caused by scattering is measured approximately $0.2 \mathrm{~dB}$. We report the statistic data of etching quality of vertical micromirrors made by TMAH as a reference. The large surface area of the micromirror can accommodate a big optical beam for 2-D matrix configuration, which will reduce the optical insertion loss caused by beam divergence. Chips consisting of $10 \times 10$ arrays are successfully fabricated and tested.

\section{REFERENCES}

[1] L. Y. Lin, E. L. Goldstein, J. M. Simmons, and R. W. Tkach, "High-density micromachined polygon optical crossconnects exploiting network connection-symmetry," IEEE Photon. Technol. Lett., vol. 10, no. 10, pp. 1425-1427, Oct. 1998.

[2] M. C. Wu, "Micromachining for optical and optoelectronic systems," Proc. IEEE, vol. 85, no. 11, pp. 1833-1856, Nov. 1997.

[3] M. Yano, "Compact and stable optical crossconnect 3D-MEMS switch and its system applications," in Proc. IEEE/LEOS Optical MEMS 2004, Aug. 2004, pp. 156-157.

[4] J. Ford, V. Aksyuk, J. Bishop, and J. Walker, "Wavelength add/drop switching using tilting micromirrors," J. Lightw. Technol., vol. 17, no. 5, pp. 904-911, May 1999.

[5] R. T. Chen, H. Nguyen, and M. C. Wu, "A high-speed low-voltage stressinduced micromachined $2 \times 2$ optical switch," IEEE Photon. Technol. Lett., vol. 11, no. 11, pp. 1396-1398, Nov. 1999.

[6] C. Marxer, C. Thio, M. Gretillat, N. F. de Rooji, R. Battig, R. Anthamatten, B. Valk, and P. Vogel, "Vertical mirrors fabricated by deep reactive ion etching for fiber-optic switching applications," J. Microelectromech. Syst., vol. 6, no. 7, pp. 277-285, Jul. 1997.

[7] L. Fan, "Micromachined Optomechanical Switching Device," U.S. Patent 6449406 B1.

[8] H. Toshiyoshi and H. Fujita, "Electrostatic micro torsion mirrors for an optical switch matrix," J. Microelectromech. Syst., vol. 5, no. 4, pp. 231-237, Dec. 1996.

[9] L. Lin, E. Goldstein, and R. Tkach, "On the expandability of the freespace micromachined optical cross connects," J. Lightw. Technol., vol. 18, no. 4, pp. 482-489, Apr. 2000.

[10] J. W. Judy, "Microelectromechanical Systems (MEMS) - Their design, fabrication, and broad range of application," J. Smart Mater, vol. 10, no. 6, pp. 1115-1134, Dec. 2001.

[11] C. Liu, T. Tsao, G. B. Lee, J. Leu, Y. Yi, Y. C. Tai, and C. M. Ho, "Out-of-plane magnetic actuators with electroplated permalloy for fluid dynamics control," Sens. Actuators A, Phys., vol. 78, no. 2-3, pp. 190-197, Dec. 1999.

[12] G. Su, H. Toshiyoshi, and M. C. Wu, "Surface-micromachined 2-D optical scanners with high-performance single-crystalline silicon micromirrors," IEEE Photon. Technol. Lett., vol. 13, no. 6, pp. 606-608, Jun. 2001.

[13] L. Houlet, P. Helin, T. Bourouina, G. Reyne, E. Gergam, and H. Fujita, "Movable vertical mirror arrays for optical microswitch matrixes and their electromagnetic actuation," IEEE J. Sel. Topics Quantum Electron., vol. 8, no. 1, pp. 58-63, Jan./Feb. 2002.

[14] N. Miki, C. Teo, L. Ho, and X. Zhang, "Precision fabrication of highspeed micro-rotors using deep reactive ion etching (DRIE)," in Proc. Solid-State Sensor, Actuator and Microsystems Workshop, Jun. 2002, pp. 265-268.

[15] E. M. Conway and V. J. Cunnane, "Electrochemical characterization of $\mathrm{Si}$ in tetra-methyl ammonium hydroxide (TMAH) and TMAH:Triton-X-100 solutions under white light effects," J. Micromech. Microeng., vol. 12, pp. 136-148, 2002.

[16] M. J. Madou, Fundamentals of Microfabrication 2nd Edition, ch. 4, pp. 214-217.

[17] F. Laermer and A. Schilp, "Method of Anisotropically Etching Silicon," U.S. Patent 5501893.

[18] O. Tabata, R. Asahi, and S. Sugiyama, "Anisotropic etching with quarternary ammonium hydroxide solutions," in Tech. Dig. 9th Sensor Symp., Tokyo, Japan, 1990, pp. 15-18. 